\title{
Rezidivprognose bei Klarzell-RCC per Multigen-Assay abschätzen
}

Multigen-Assays liefern validierte prognostische Informationen, die die klassischen Prognosefaktoren ergänzen können. Für resektable Klarzell-Nierenzellkarzinome wurde jetzt ein genprofilbasierter Rezidiv-Risikoscore entwickelt.

Ü ber $80 \%$ der sporadisch auftretenden Nierenzellkarzinome (RCC) vom Klarzelltyp weisen Fehlregulationen in der von-Hippel-Lindau-Signaltransduktion und den dazugehörigen Genen für den Hypoxie-induzierten Faktor auf. Forscher suchten nun nach einer Gensignatur beim RCC, mit dem sich die Rezidivwahrscheinlichkeit vorhersagen lässt.

Dazu analysierten sie die Assoziation zwischen dem Expressionsprofil von 732 Genen und dem klinischen Ergebnis von 942 RCC-Patienten im Stadium I-III, bei denen eine Nephrektomie ohne weitere adjuvante Therapie erfolgte. Die mediane Beobachtungszeit betrug 6,2 Jahre. Zunächst wurden 516 mit Rezidivfreiheit assoziierte Gene identifiziert. Nach weiteren statistischen Modellierungen zusammen mit 5 Referenzgenen ein Rezidivscore-Algorithmus entwickelt und dieser an einer neuen, unabhängigen Kohorte von 626 nephrektomierten RCC-Patienten im Stadium I-III validiert wurde.

In der univariaten Analyse zeigte der neue Rezidivscore tatsächlich eine hochsignifikante Assoziation mit dem rezidivfreien Intervall (Hazard Ratio [HR] pro Anstieg um 25 Scoreeinheiten 3,91; $95 \%$-Konfidenzintervall [95\%-KI] 2,635,79; $\mathrm{p}<0$ 0001). Auch nach stadienspezifischer Stratifizierung und Adjustierung auf Tumorgröße und -grad sowie den Leibovich-Score blieb der signifikanfiel die Wahl auf 11 Gene, für die dann te Zusammenhang erhalten (HR pro Anstieg um 25 Scoreeinheiten 3,37; $95 \%$-KI 2,23-5,08; $\mathrm{p}<0,0001)$. Hochrisikopatienten im Stadium I sowie Niedrigrisikopatienten im Stadium II-III identifizierte der neue Rezidivscore in klinisch akzeptablem Umfang. Heterogenitätstests an weiteren Tumorproben zeigten nur eine geringe bis gar keine intratumorale Variabilität bei den ausgewählten Genen.

Fazit: Ein neuer, genbasierter Risikoscore erlaubt eine über die klassische Risikoeinschätzung hinausgehende Rezidiv-Risikovorhersage bei RCC-Patienten mit Klarzelltumoren im Stadium IIII. Dadurch lassen sich adjuvante Therapien noch stärker als bisher individualisieren.

Barbara Kreutzkamp

Rini B et al. A 16-gene assay to predict recurrence after surgery in localised renal cell carcinoma: development and validation studies. Lancet Oncol. 2015;16(6):676-85.

\section{Zytokin-aktivierte Killerzellen beim RCC wirksam und sicher}

Die Immuntherapie mit patienteneigenen, Zytokin-aktivierten Killerzellen - mit oder ohne tumorlysatgetriggerte dendritische Zellen verbessert Überlebensparameter von RCC-Patienten bei guter Sicherheit, so das Ergebnis einer Proof-of-Concept-Studie.

mmunologische Vorgänge spielen in Entwicklung und Verlauf von Nierenzellkarzinomen (RCC) eine wichtige Rolle. Therapeutisch wird dieses Wissen bisher kaum umgesetzt. Einen interessanten Ansatz bieten Zytokin-induzierte Killerzellen (CIK). Eingesetzt werden vor allem durch Zytokine wie Interleukin(IL)-2 aktivierte CD3-/ CD56-doppelpositive T-Lymphozyten, entnommen aus dem peripheren Blut der Patienten. Um die Therapieeffizienz weiter zu steigern, kultiviert man CIK zusammen mit dendritischen Zellen, die zuvor durch autologes Tumorlysat getriggert wurden (Ag-DC-CIK).

In einer monozentrisch und einfachblind durchgeführten Studie sollte nun untersucht werden, wie RCC in verschiedenen Stadien auf die zellbasierte Im- muntherapie reagieren. Dazu erhielten insgesamt 60 operierte Patienten randomisiert eine Zellsuspension mit den aktivierten Ag-DC-CIK, gegeben in 6 jeweils 3-tägigen Infusionszyklen alle 15 Tage oder die Standardtherapie. Die 62 nicht operablen Patienten erhielten CIK oder die stadiengerechte Standardbehandlung.

Bei den operierten Patienten betrug die Rate für das krankheitsfreie Überleben (DFS) nach 3 Jahren unter Ag-DC-CIK 96,7\% verglichen mit 57,7\% in der Kontrollgruppe ohne Immuntherapie ( $p=0,0418)$. Bei den inoperablen Patienten betrug die Rate für das Gesamtüberleben (OS) nach 3 Jahren 48,8\% im Vergleich zu 21,2\% in der Kontrollgruppe $(\mathrm{p}=0,0116)$, das mediane OS war mit 28 versus 11 Monaten deutlich verlängert.
Entsprechende Unterschiede ergaben sich auch beim progressionsfreien Überleben nach 3 Jahren. Das CD4 ${ }^{+} / \mathrm{CD}^{+}{ }^{+}$ZZellverhältnis im peripheren Blut, gemessen nach der letzten Infusion, stieg unter CIK und - noch stärker - unter den Ag-DCCIK im Vergleich zur Kontrollgruppe an. Schwere Toxizitäten wurden nicht gemeldet. Häufigste Nebenwirkung waren grippeähnliche Symptome, die sich 2 bis 4 Stunden post infusionem ohne weitere Behandlung rasch besserten.

Fazit: Eine Therapie mit Zytokin-induzierten Killerzellen bietet RCC-Patienten eine gut verträgliche Therapieoption. Nicht mehr operable Patienten profitieren deutlich, bei operierten Patienten bietet sich eine noch effektivere adjuvante Behandlung mit CIK plus tumorlysatgetriggerten DC an. Barbara Kreutzkamp

Zhao X et al. Cytokine induced killer cell-based immunotherapies in patients with different stages of renal cell carcinoma. Cancer Lett. 2015;362(2):192-8. 